\title{
UN ESTUDIO SOBRE EL MÉTODO ESTITSOLÓGICO MULTISENSORIAL DE ATENCIÓN TEMPRANA ${ }^{1}$
}

\author{
A STUDY ON THE EARLY ATTENTION ESTITSOLOGIC \\ MULTI-SENSORIAL METED
}

\author{
Pilar Ibáñez López*, María José Mudarra Sánchez** y Cristina Alfonso Ibáñez*** \\ Universidad Nacional de Educación a Distancia (UNED)
}

\section{RESUMEN}

Dentro de la línea de investigación que estamos realizando sobre estimulación temprana, con la aplicación del Método Multisensorial, es este artículo se presenta un estudio sobre dicho método, en una muestra de 478 niños que viven en contextos normalizados, de 0 a 6 años. Se valora el impacto de su aplicación - fundamentalmente por los padres - durante cuatro meses, analizando la significatividad de sus efectos en distintas áreas de desarrollo (socio-afectivo, perceptivo-cognitivo, motriz, pensamiento y lenguaje), considerando la influencia de variables como la edad del niño y de sus padres y el número de hermanos. Se sigue un diseño pretest-postest con grupos experimental y control, en el que se analizan los datos mediante diversos procedimientos estadísticos ( $t$ de Student, ANCOVA, medidas de asociación etc.). Los resultados obtenidos demuestran la eficacia del Método Estitsológico Multisensorial en todas las dimensiones de desarrollo, especialmente en el desarrollo socio-afectivo y la coordinación sensomotriz, que justifican su aplicación como una estrategia eficaz de estimulación temprana.

Palabras clave: Atención temprana, estimulación, Método Estitsológico Multisensorial, estudio empírico, análisis estadístico.

* Pilar Ibáñez López. Profesora Titular del Departamento MIDE II, Facultad de Educación, UNED. Autora de numerosos libros y artículos, su línea de investigación actual: poblaciones especiales y estimulación temprana.

** María José Mudarra Sánchez Dra. en Filosofía y Ciencias de la Educación por la UNED. Licenciada en Filosofía y Ciencias de la Educación por la U.C.M., Profesora colaboradora en el Dpto. MIDE II, Facultad de Educación, UNED. Líneas de investigación: Orientación para el Desarrollo de la Carrera, Discapacidad y Métodos de investigación educativa.E-mail Mjosemud@ya.com.

*** Cristina Alfonso Ibáñez. Dra. en Filología Inglesa, experta en Nuevas Tecnologías. Líneas de investigación: aprendizaje de idiomas en la primera infancia y Literatura en Lengua Inglesa. Profesora Tutora de la UNED.

${ }^{1}$ Desde aquí expresamos nuestro agradecimiento a la Org. Mundial de Ed. Preescolar (entidad consultiva ante UNESCO, UNICEF y Consejo de Europa) y al Dpto. MIDE II (Orient. Educativa, Diag. e Intervención Psicopedagógica), por su apoyo en la realización del presente estudio. 


\begin{abstract}
In this article we present a study within the line of investigation which we are developing on early stimulation, (with the application of the Multisensorial Estitsologic Method), with a sample of 478 children of 0 to 6 years old all of whom live in standardized contexts. The impact of its application is valued during four months, during which we analyze the significance of its effects in different areas of development (socio-affective, perceptive-cognitive, motor, thought and language). An experimental pretest-postest design is followed with the use of experimental and control groups in which the data are analyzed by means of diverse statistical procedures ( $t$ of Student, ANCOVA, grade of association etc.). The obtained results demonstrate the effectiveness of the Multisensorial Estitsologic Method in all the dimensions of development, specially in the socio-affective development and the coordination, that justify its application as an effective strategy for early stimulation.
\end{abstract}

Key words: Early stimulation, Multisensorial Estitsologic Method, empiric study, statistical analysis.

\title{
Introducción
}

Nuestra extensa trayectoria profesional sobre personas con discapacidad psíquica influyó en el interés por la atención temprana, iniciando hace tiempo una línea de investigación que ha permitido proponer diversos modelos de estimulación a través de cuidados habituales al niño, teniendo en cuenta cuáles eran los ejercicios más eficaces, sencillos y breves de aplicar a niños con o sin necesidades educativas especiales. A retomar la trayectoria de investigación en Estimulación nos impulsaron razones tales como las abundantes cifras de maltrato físico y psicológico que están sufriendo cierto número de niños — preocupante en una sociedad cada vez más industrializada-, las peleas en el hogar, la incomunicación entre padres e hijos, así como otros factores que impulsan a muchos jóvenes a buscar refugio extremo en las drogas y a aislarse emocionalmente buscando una vía de escape. Se piensa que esas situaciones tienen su origen en fallos en el establecimiento o fortalecimiento de vínculos afectivos entre padres e hijos que ocasionan alteraciones emocionales en la personalidad de los niños muchas de las cuales permanecen o dejan secuelas durante toda la vida del individuo, tanto de tipo psíquico o psicológico como a veces físico. Por ello preparar para la vida implica la estimulación para el desarrollo de estrategias de aprendizaje autónomo - permanente - que, en línea con los planteamientos actuales sobre inteligencia emocional, preparen al niño para que tenga estabilidad emocional y seguridad en sí mismo, tome decisiones responsables y se relacione satisfactoriamente con los demás.

Para paliar las situaciones anteriormente mencionadas, hace algunos años se lanzaron eslóganes tales como "habla con tu hijo" y actualmente la mayoría de los pediatras recomiendan a los padres que hablen y jueguen con sus hijos desde la más temprana edad para que emocionalmente crezcan sanos. En respuesta a las demandas sociales en pro de la infancia, y con la finalidad de potenciar los vínculos afectivos entre padres e hijos al mismo tiempo que se produce un efecto de estimulación multisensorial en los niños - como se ha manifestado en varios estudios realizados, entre los que destacamos el referido a madres con sus bebés en prisión (Ibáñez y Alfonso, 1999; Ibáñez y Senra, 1999) — hemos iniciado acciones e investigaciones, utilizando como instrumento principal de trabajo el Método Estitsológico Multisensorial (Ibáñez 1997 y 2002), surgido del estudio y análisis de técnicas aplicadas - en varios países - a bebés, con predominio del uso del tacto.

La importancia de que el niño se sienta emocionalmente seguro ha sido reconocida por Organismos tales como Las Naciones Unidas que, en su Asamblea General convocada en 
mayo de 2002, celebró una Sesión Especial a favor de la Infancia aprobando por consenso el documento Un Mundo apropiado para los Niños, convertido a partir de entonces en guía esencial para quienes trabajan con ellos en todo el mundo. De los nueve principios formulados en dicho documento, destacamos el principio 7.4 cuyo título es Cuidar de todos los niños, en él encontramos la prioridad de "criar a los niños en un entorno seguro que les permita disfrutar de buena salud, estar mentalmente alerta, sentirse emocionalmente seguros y ser socialmente competentes y capaces de aprender". Y esos cuidados que precisa el niño para el pleno y armonioso desarrollo, deben ser procurados por la familia para que crezca en un ambiente de felicidad, amor y comprensión (Convención sobre los Derechos del Niño, 1989). Cuanto antes se inicien estos cuidados los resultados serán más satisfactorios y además se estará poniendo en práctica el carácter preventivo ante la aparición de ciertos problemas; incluso en la Declaración de los Derechos del Niño, 1924, se señala que el niño necesita además de atención postnatal una atención prenatal. Indudablemente que los requerimientos de todo tipo de atención comienzan por el aspecto médico y la protección legal pero actualmente se empieza a reconocer la necesidad e influencia de los cuidados emocionales a la embarazada y al hijo cuando está en el útero (Ibáñez, 2002).

\section{La estimulación temprana del desarrollo infantil}

El término "Estimulación Temprana" se acuñó para indicar la estimulación programada que el niño recibe casi desde su nacimiento y durante sus primeros años de vida, esto es, un tratamiento con bases científicas para desarrollar al máximo sus posibilidades psicofísicas, (Ibáñez, 2002). Aplicable a cualquier niño con o sin alteraciones —en sí mismo y/o en su contexto familiar - resulta fundamental para prevenir y/o compensar discapacidades psíquicas, físicas, sensoriales, de privaciones afectivas o alteraciones emocionales.

El desarrollo infantil es un proceso dinámico, sumamente complejo, fruto de la interacción entre factores genéticos y ambientales, que se sustenta en la evolución biológica, psicológica y social, dando como resultado la maduración orgánica y funcional del sistema nervioso, el desarrollo de funciones psíquicas y la estructura de la personalidad. Luego, a partir de la base genética de cada persona que confiere ciertas capacidades propias de desarrollo, son los factores ambientales los que condicionan en mayor o menor grado, la expresión de estas capacidades. Efectivamente, el conocimiento del entorno en el que se desenvuelve el niño es fundamental para detectar factores potenciadores o inhibidores de su desarrollo, de ahí que modelos como la $\mathrm{T}^{\mathrm{a}}$ General de Sistemas y el enfoque ecosistémico, resulten útiles para explicar la eficacia de estas intervenciones al considerar a la familia como un sistema funcional donde se suceden múltiples modalidades de interacción.

En la interacción entre la familia y el niño, el rol de los padres como mediadores o terapeutas que facilitan el desarrollo de sus hijos, participando activamente en los programas de estimulación y educación tempranas, ha resultado fundamental para explicar los progresos y su continuidad en el tiempo, tal como demostró Brofenbrenner (1975), en su importante revisión de numerosos programas de intervención. Heward (1998) señala dos variables que parecen estar íntimamente relacionadas con la eficacia de la intervención temprana: la intensidad de la intervención y el nivel de participación de las personas implicadas en los programas, de ahí la importancia de implicar a los padres en este tipo de interacciones con sus hijos puesto que son los más interesados en promover su desarrollo y los que disponen de 
más oportunidades para participar activamente en su estimulación. Los vínculos afectivos que el niño establece a partir del afecto y estabilidad en los cuidados que recibe, la percepción de cuanto le rodea, son necesidades básicas del ser humano y determinantes de su desarrollo emocional, funciones comunicativas, conductas adaptativas y actitudes hacia el aprendizaje. La propia carencia o limitación del contacto con los padres y/o una estimulación social inadecuada, constituyen factores de riesgo psico-afectivo.

Los primeros años de vida constituyen una etapa de la existencia especialmente crítica ya que en ella se van a configurar las habilidades perceptivas, motrices, cognitivas, lingüísticas, afectivas y sociales que posibilitarán una equilibrada interacción con el mundo circundante. Así, se adquieren progresivamente funciones tan importantes como el control postural, la autonomía de desplazamiento, la comunicación, el lenguaje verbal y la interacción social, (Real Patronato sobre Discapacidad, 2003).

La Atención Temprana trata de ofrecer - a través de distintos ámbitos como la Sanidad, Servicios Sociales y Educación - un conjunto de acciones que faciliten la adecuada maduración en todos los ámbitos, que permita alcanzar el máximo nivel de desarrollo personal y de integración social. En este proceso integral de desarrollo armónico de los niños integrados en su entorno, además del diagnóstico precoz de factores de riesgo, se enfatizan los procesos optimizadores de dicho desarrollo y la adopción de medidas preventivas. Por otra parte, se reconoce la rentabilidad económica, social y personal de la Atención Temprana - muchas alteraciones de desarrollo pueden prevenirse si son tratadas a tiempo, promoviendo con ello el bienestar social de las familias y el de la sociedad en su conjunto-, ya que factores como la dedicación afectiva, la estabilidad de las relaciones familiares, la suficiencia económica, la estabilidad laboral, la participación en redes sociales y la coherencia de los estilos educativos, son fundamentales en el desarrollo infantil.

\section{El Método Estitsológico Multisensorial}

Su denominación hace referencia al término griego stitso — así como los latinos "stimulus" y "stimulare"-. A través de los sentidos, la realidad llega a nuestro cerebro y éste a su vez, construye de nuevo el entorno, mediante reinterpretaciones sucesivas. El Método Estitsológico Multisensorial, en cuya aplicación debe darse preferencia a la familia — padre-madre- por el fortalecimiento o incremento de los vínculos afectivos entre ambos (aplicador y niño), desarrolla la percepción sensorial del niño y los procesos cognitivos y motrices por medio de la estimulación multisensorial, teniendo por base técnicas de estimulación a través del tacto.

A continuación, presentamos (ver figura 1) la Ficha técnica del Método Estitsológico, especialmente apreciado por los padres debido a la flexibilidad tanto en la aplicación del número de sesiones semanales como en su duración temporal - lo que reduce el abandono de su práctica al poder compaginarlo fácilmente con la actividad diaria, sin necesidad de trabajos preparatorios o tiempos específicos- y por la capacidad de observación que se desarrolla en el aplicador. Así pues, además de favorecer los sentimientos de empatía padre/madre con los hijos, se aprecia la Ley del Efecto de Thorndike — la repetición de una conducta depende de sus consecuencias - y la Ley de Jost — aprendizaje fraccionado mediante pausas en el curso del aprendizaje, acomodación del ritmo de las estimulacionesincidiendo en los aspectos motrices, cognitivos y emocionales, acelerando el proceso de 
evolución de los bebés — gatear, andar- e incrementando los vínculos afectivos niñoaplicador.

\section{FICHA TÉCNICA}

- Población a la que va dirigida: Niños con/sin discapacidad que viven en ambientes normalizados o con algún tipo de alteración. Aplicable a niños desde el nacimiento.

- Objetivos generales que pretende alcanzar:

Para el niño:

- Afianzar los vínculos afectivos con la persona/s que le aplican el método, fomentando la comunicación entre ambos. Adquirir seguridad, confianza e independencia.

- Ayudarle a relajarse corporalmente, para crear hábito.

- Estimular los sist. nervioso y muscular, a través de la sensibilidad cutánea

- Intervenir en la evolución de los procesos cognitivos a través de la estimulación multisensorial y motriz

Para el aplicador:

- Adquirir el hábito de liberarse de preocupaciones y dedicar algún tiempo a los hijos
- Conocer mejor al hijo aprendiendo a interpretar su lenguaje corporal

- Adquirir autoconfianza en el trato con el niño

- Promover el sentimiento de cuidado al hijo.

- Bloques de actividades:

I. Preparación para la aplicación del Método.

II. Piernas y pies/brazos y manos.

III. Tronco.

IV. Cara, orejas y cuello.

V. Cráneo, cuello, espalda y nalgas.

(Cada bloque posee objetivos específicos).

- Materiales necesarios: Toallas —para situar y limpiar al niño-, aceite vegetal y un recipiente para verterlo.

- Frecuencia de las sesiones: Flexible. Número de sesiones en función la disponibilidad del aplicador.

- Duración de las sesiones: Flexible. Aprox. 20 min. si se aplica en todo el cuerpo.

\section{FIGURA 1.}

Ficha técnica del Método Estitsológico.

Este Método ha sido validado a través de diversos expertos, profesionales especialistas en pediatría y salud pública, educación y psicología infantil, orientación educativa y terapéutica, quienes valoraron los bloques de actividades, su pertinencia respecto a los objetivos propuestos, la claridad y expresión del lenguaje empleado y la adecuación del número de actividades propuestas; se realizó una valoración global de la importancia de estos bloques, sus limitaciones, estructura y extensión. Para comprobar su eficacia, se han realizado estudios longitudinales sobre diversas poblaciones y estudio de casos en profundidad, en los que se han obtenido resultados positivos, (Ibáñez, 2003; Ibáñez, 1997; Ibáñez y Alfonso, 1999). En particular, cabe destacar el estudio del método sobre muestras de bebés nacidos en la cárcel que compartían con sus madres el mismo espacio ambiental. Tras aplicarlo durante nueve meses, para compensar las carencias derivadas de este ambiente desfavorecido, el análisis de la evolución de sus cocientes de desarrollo general antes y después de di- 
cha aplicación, resultó ser muy positivo y significativo. Además, se constató que los niños cuyo embarazo había sido deseado presentaban cocientes de desarrollo más elevados, siendo superior su desarrollo motriz y de lenguaje (Ibáñez y Senra, 1999a). También la actitud positiva de la madre ante el embarazo se relacionaba positiva y significativamente con el cociente general de desarrollo y en particular, con el desarrollo motriz (Ibáñez y Senra, 1999b). Paralelamente, hallamos efectos positivos y significativos en muestras de hijos de alcohólicos y niños con discapacidades (Ibáñez y col. Inédito).

\section{Diseño del estudio}

\section{Objetivo General}

El objetivo general de este estudio es valorar el impacto de la aplicación del Método Estitsológico Multisensorial en niños sin problemas detectados, que viven en contextos normalizados, esto es, analizar la significatividad de sus efectos en distintas áreas de desarrollo (socio-afectivo, perceptivo-cognitivo, motriz y de pensamiento-lenguaje) y la posible influencia en su desarrollo de variables como la edad del niño y de sus padres y el número de hermanos.

\section{Cuestiones}

Así, nos planteamos las siguientes cuestiones de investigación:

1. ¿Cuál es la evolución socio-afectiva, perceptivo-cognitiva, motriz, de pensamiento y lenguaje, tras 4 meses, tanto en los niños a los que se les aplica el método - grupo experimental - como en los niños del grupo control? (Efectos dentro de los grupos).

2. Teniendo en cuenta que dentro de un grupo ciertos niños pueden progresar significativamente, a pesar de que el grupo en su conjunto no manifieste dicho progreso, nos planteamos cuál sería el ritmo individual de su desarrollo, en ambos grupos y en cada una de las citadas áreas, teniendo en cuenta su edad, ¿cuántos manifiestan aceleración, estancamiento o siguen un ritmo de desarrollo normalizado — conforme las escalas consideradas-? (Efectos individuales).

3. La aplicación del Método Estitsológico Multisensorial, ¿produce efectos significativos en el desarrollo global de los niños, esto es, en las áreas de desarrollo socioafectivo, perceptivo-cognitivo, motriz y de pensamiento-lenguaje? (Efectos entre los grupos). En particular,

3.1. ¿Son significativas las diferencias en cada una de estas áreas entre los grupos experimental y control, debidas a la aplicación del método, teniendo en cuenta su nivel inicial de desarrollo? — efecto principal del método-.

3.2. ¿Son significativas las diferencias obtenidas en cada área de desarrollo — socioafectivo, perceptivo-cognitivo, motriz, de pensamiento y lenguaje - tras la aplicación del método debidas a la edad de los sujetos? - efecto principal de la edad-. 
3.3. El nivel inicial de cada área de desarrollo — socio-afectivo, perceptivo-cognitivo, motriz, de pensamiento y lenguaje - ¿influye significativamente en los resultados obtenidos en dichas áreas tras la aplicación del método? —efecto principal del pretest-.

3.4. ¿Existen diferencias significativas en cada una de las áreas de desarrollo - socio-afectivo, perceptivo-cognitivo, motriz, de pensamiento y lenguajeentre el grupo experimental y el de control, en los sub-grupos establecidos según la edad y los niveles de desarrollo inicial de los sujetos en cada una de las áreas, por efecto del método? —efecto de interacción método $\mathbf{x}$ edad $\mathbf{x}$ pretest-.

3.5. El tamaño del efecto del tratamiento sobre cada una de las áreas de desarrollo - socio-afectivo, perceptivo-cognitivo, motriz, de pensamiento y lenguaje¿justifica su aplicación?

4. ¿Se relacionan significativamente la edad de los padres y el número de hermanos que reciben estos niños con su nivel de desarrollo afectivo, perceptivo-cognitivo, motriz, de pensamiento y lenguaje?

\section{Hipótesis y Variables}

Para responder a las cuestiones planteadas, fue preciso comprobar las siguientes hipótesis sustantivas:

1. Tras los cuatro meses del estudio, deberían apreciarse cambios significativos en los niveles de desarrollo socio-afectivo, perceptivo-cognitivo, motriz, de pensamiento y lenguaje de los niños cualquiera que fuese el grupo al que fueron asignados - experimental y control- (efectos dentro de los grupos).

2. La aplicación del Método Estitsológico Multisensorial produce mejoras significativas en los niveles de desarrollo socio-afectivo, perceptivo-cognitivo, motriz, pensamiento y lenguaje - considerando influencias debidas a su edad y nivel de desarrollo previo- que justifican su aplicación.

3. Existen relaciones significativas entre la edad de los padres y el nivel de desarrollo que alcanzan sus hijos en las distintas áreas.

4. Existen relaciones significativas entre el número de hermanos de los niños y el nivel de desarrollo que alcanzan en las distintas áreas.

Por tanto, consideramos las siguientes variables:

- Variable independiente: el Método o tratamiento aplicado a los niños asignados al grupo experimental.

- Variables dependientes: cada una de las dimensiones que integran las distintas áreas de desarrollo (ver tabla 1) cuyos valores se registran tras cuatro meses de aplicación del método (Postest).

- Variables Intervinientes: se controla tanto el Pretest, esto es, el nivel inicial en cada una de las dimensiones de desarrollo, como la Edad. 
TABLA 1: Áreas de desarrollo evaluadas.

\begin{tabular}{|l|l|l|}
\hline \multicolumn{1}{|c|}{ Áreas de Desarrollo } & \multicolumn{1}{|c|}{ Comprende } & \multicolumn{1}{c|}{ Dimensiones evaluadas } \\
\hline Socio-afectivo & $\begin{array}{l}\text { Habilidades y actitudes del niño } \\
\text { frente a su medio socio-cultural }\end{array}$ & Afectividad (Af) \\
\hline Perceptivo-cognitivo & $\begin{array}{l}\text { Ajustes sensomotores frente a } \\
\text { objetos y situaciones } \\
\text {-discriminación y adquisición } \\
\text { de conocimientos-. }\end{array}$ & $\begin{array}{l}\text { Desarrollo Somático (som) } \\
\text { Desarrollo Senso-perceptivo (sens) }\end{array}$ \\
\hline Motriz & $\begin{array}{l}\text { Movimientos corporales, } \\
\text { coordinaciones motrices }\end{array}$ & $\begin{array}{l}\text { Reacción Motriz (mot) } \\
\text { Coordinación Senso-Motriz (sm) }\end{array}$ \\
\hline Pensamiento y lenguaje & $\begin{array}{l}\text { Toda forma de comunicación, } \\
\text { conceptuación, seguimiento de } \\
\text { reglas y tareas }\end{array}$ & $\begin{array}{l}\text { Contacto y Comunicación (com) } \\
\text { Conceptualización (cpto) } \\
\text { Normatividad (nor) }\end{array}$ \\
\hline
\end{tabular}

\section{Instrumento de Evaluación del Desarrollo}

Existen numerosas escalas de desarrollo en el mercado que suelen explorar las áreas motora, perceptivo-cognitiva, de lenguaje y social - aunque reciban diversas denominaciones - para determinar los niveles de madurez a partir de los cuáles interpretar el desarrollo del niño. Bajo el supuesto de que el desarrollo se expresa externamente por las reacciones reflejas, voluntarias, espontáneas y aprendidas, éste se puede examinar a través de la exploración de los "modos de conducta".

En el presente estudio, tanto la evaluación inicial - pretest- como la evaluación de cada área de desarrollo tras la aplicación del Método — postest — se llevó a cabo mediante la Escala Observacional del Desarrollo, (Secadas, 1988), por ser un instrumento fácil de aplicar, accesible y ampliamente validado con muestras semejantes a la nuestra. Tomando como referencia intervalos de tiempo, diagnostica el estado de desarrollo en que se encuentran los niños en cada una de las dimensiones citadas anteriormente, fomentando la observación de conductas - tanto por el aplicador como por los padres - cuya presencia/ausencia determina los perfiles de desarrollo general.

\section{Muestra de sujetos}

La muestra está formada por 478 niños, procedentes de diversas comunidades autónomas, de 0 a 6 años, sin ningún tipo de alteración o discapacidad diagnosticada y que viven en contextos normalizados. Respecto a las características de la muestra, destacamos los siguientes aspectos:

- Se trata de una muestra equivalente respecto al género - conforme la prueba binomial一, 268 varones, $(56,1 \%)$ y 210 mujeres $(43,9 \%)$.

- Se distribuye uniformemente respecto a la edad, concentrándose la mayor parte de la muestra en edades comprendidas entre 1 y 5 años. 
- No existe una relación significativa entre el género y la edad de los sujetos (Chi-cuadrado $=3,678$, Sig. $=0,816$ ), obteniéndose la distribución de género en función de la edad que puede observarse en la figura 2.

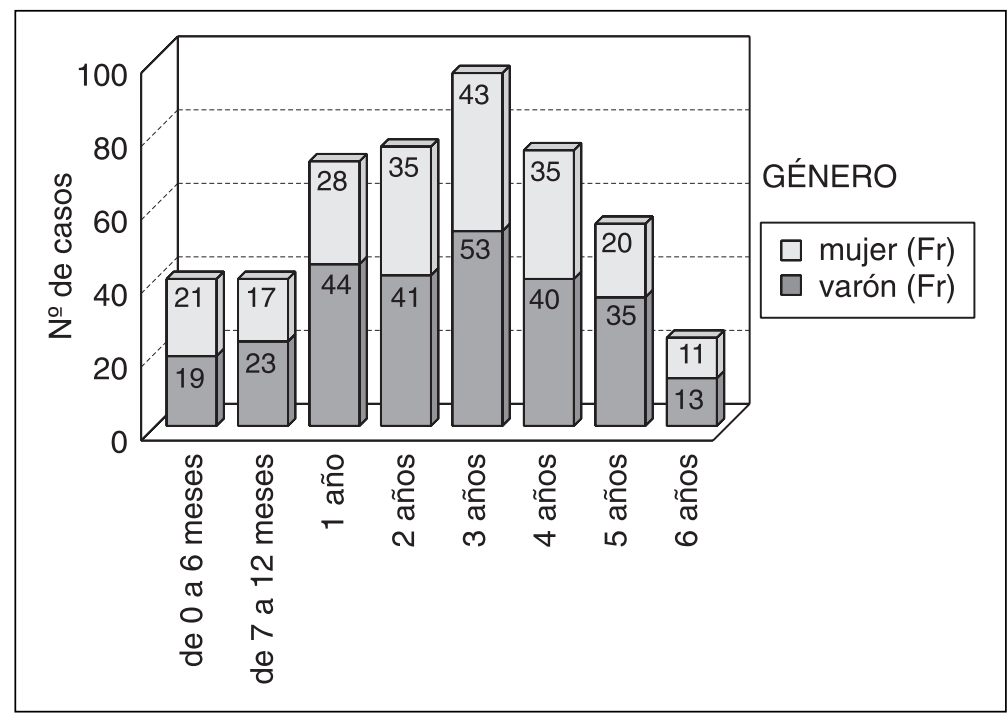

FIGURA 2.

Distribución del género en función de la edad.

- La edad media del padre está en torno a los 34 años y de la madre entorno a los 31 años, mientras que las edades medias de los hermanos oscilan entre los 7 y los 9 años (ver figura 3).

- Predominan los sujetos con un solo hermano o hermana $(77,4 \%$ y $84,4 \%)$ y en menor medida tienen dos o tres hermanos (22,5\% y 15,6\%, respectivamente) (ver figura 4$)$.

\section{Procedimiento de aplicación del método}

Se siguió el procedimiento pretest-postest, aplicando la Escala Observacional del Desarrollo inicialmente para conocer el estado de desarrollo de cada niño antes de aplicar el Método - pretest - . A continuación, los padres de los niños del grupo experimental, comenzaron las sesiones de aplicación del Método durante cuatro meses, período que no se prolongó por razones éticas, esto es, privar a los niños del grupo control de los posibles beneficios que podría reportarles la aplicación del método. Finalizado el período de aplicación, se evaluó - empleando nuevamente la Escala Observacional del Desarrollo - el progreso de cada niño - postest - . La recogida de datos comparados de ambos grupos - experimental y control- sobre la evolución del cociente general del desarrollo antes y después de la aplicación del método y su posterior tratamiento estadístico, permitiría determinar la eficacia de 


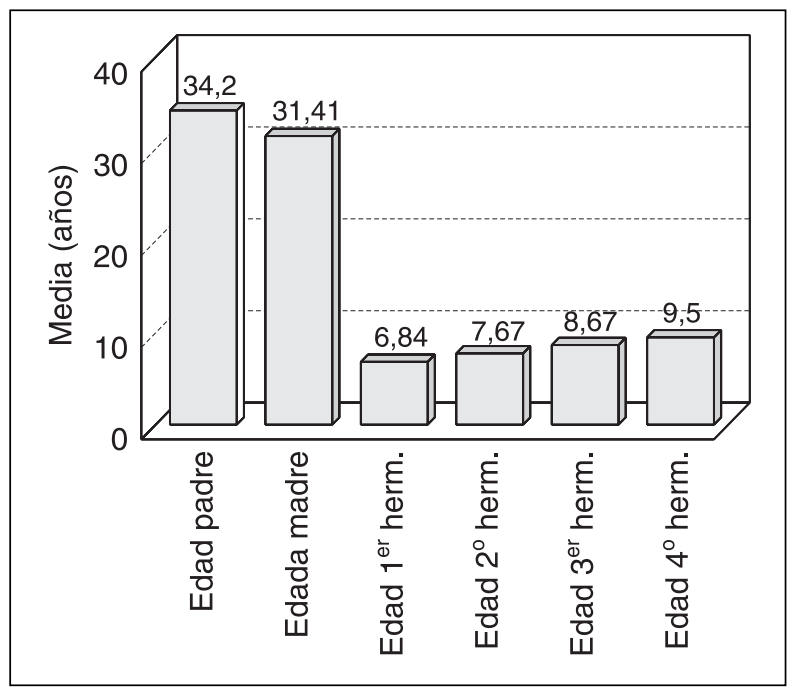

FIGURA 3.

Edad media de padres y hermanos.

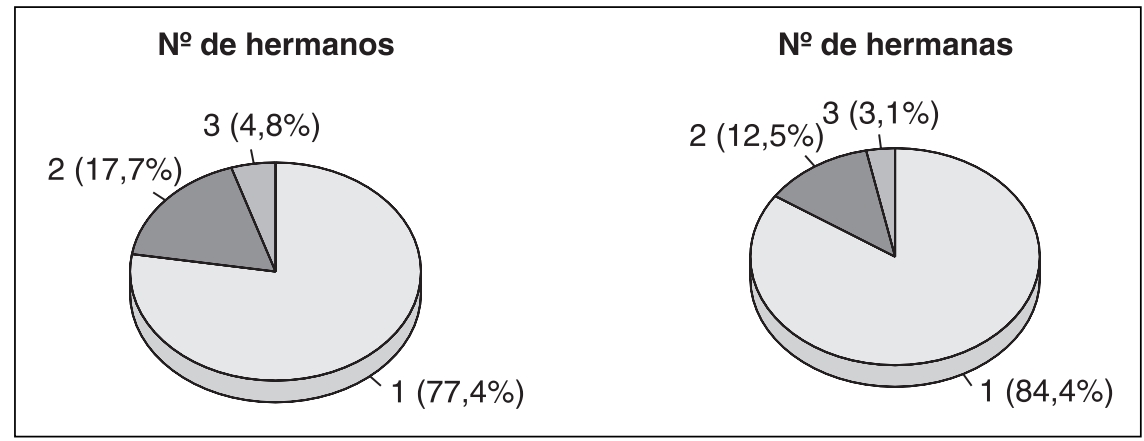

FIGURA 4.

Número de hermanos y hermanas.

dicho método. Finalizado el estudio, se instruía a los padres de los niños del grupo control en la aplicación del método para que también pudieran beneficiarse de él.

Así, el gráfico correspondiente al diseño de nuestro estudio sería:

$$
\begin{array}{lll}
\text { Pretest } & \mathrm{X} & \text { Postest (Gr. Experimental) } \\
\text { Pretest } & - & \text { Postest (Gr. Control) }
\end{array}
$$

Donde X representa la aplicación del Método Estitsológico Multisensorial. 


\section{Análisis de datos}

La metodología empleada para analizar los datos de este estudio fue básicamente cuantitativa, realizando análisis estadísticos de diferencias de medias $-t$ de student,$- \mathrm{y}$ análisis de covarianza, con ayuda del programa SPSS. Los resultados obtenidos serán complementados con análisis posteriores - de carácter más cualitativo, observacional— que se están llevando a cabo como parte de los diversos estudios que conforman esta línea de investigación sobre el Método Estitsológico Multisensorial.

Efectos del método dentro de los grupos. Evolución del desarrollo de los niños del grupo experimental y control, transcurridos 4 meses

El análisis del impacto de la aplicación de este Método en cada uno de los grupos, se llevó a cabo mediante la prueba $t$ de Student y el estudio de las medias y desviaciones típicas del grupo control y del grupo experimental, tanto en el pretest como en el postest, tomando como referencia niveles de significación menores o iguales a 0,05 (ver Tabla 2).

TABLA 2: Efectos del Método dentro de los Grupos ( $t$ de Student).

\begin{tabular}{|c|c|c|c|c|c|c|c|c|c|c|c|}
\hline \multicolumn{12}{|c|}{ EFECTOS INTRA-GRUPOS } \\
\hline \multirow{2}{*}{\multicolumn{2}{|c|}{$\begin{array}{l}\text { Cociente de } \\
\text { Desarr. }\end{array}$}} & \multicolumn{2}{|c|}{ Afectividad } & \multicolumn{2}{|c|}{ Somático } & \multicolumn{2}{|c|}{ Senso-perc. } & \multicolumn{2}{|c|}{ R. Motriz } & \multicolumn{2}{|c|}{ C. Senso-Mot } \\
\hline & & $\bar{x}$ & $S$ & $\overline{\boldsymbol{x}}$ & $S$ & $\overline{\boldsymbol{x}}$ & $S$ & $\overline{\boldsymbol{x}}$ & $S$ & $\overline{\boldsymbol{x}}$ & $S$ \\
\hline \multirow{3}{*}{$\begin{array}{c}\text { GR. } \\
\text { CTROL }\end{array}$} & Pretest & 57,69 & 23,125 & 53,15 & 25,871 & 65,23 & 28,612 & 55,38 & 25,145 & 60,09 & 24,481 \\
\hline & Postest & 68,68 & 20,582 & 71,85 & 22,461 & 78,59 & 23,514 & 71,96 & 22,941 & 77,05 & 19,496 \\
\hline & $t$ & \multicolumn{2}{|c|}{$-7,631 * * *$} & \multicolumn{2}{|c|}{$-11,089 * * *$} & \multicolumn{2}{|c|}{$-8,768 * * *$} & \multicolumn{2}{|c|}{$-10,401 * * *$} & \multicolumn{2}{|c|}{$-12,115 * * *$} \\
\hline \multirow{3}{*}{$\begin{array}{c}\text { GR. } \\
\text { EXPER. }\end{array}$} & $\mathrm{P}$ & 58,91 &, 501 & 52,79 & 25,1 & 68,34 & 25 , & 59,47 & 54, & 61,90 & 23,493 \\
\hline & Postest & 75,66 & 20,529 & 81,25 & 19,072 & 87,11 & 19,743 & 83,48 & 21,224 & 86,34 & 16,742 \\
\hline & $t$ & \multicolumn{2}{|c|}{$-11,076^{* * *}$} & \multicolumn{2}{|c|}{$-17,104 * * *$} & \multicolumn{2}{|c|}{$-12,486 * * *$} & \multicolumn{2}{|c|}{$-6,852 * * *$} & \multicolumn{2}{|c|}{$-15,333 * * *$} \\
\hline
\end{tabular}

\begin{tabular}{|c|c|c|c|c|c|c|c|}
\hline \multirow{2}{*}{\multicolumn{2}{|c|}{ Cociente de Desarr. }} & \multicolumn{2}{|c|}{ Contacto-Comunica. } & \multicolumn{2}{|c|}{ Conceptualización } & \multicolumn{2}{|c|}{ Normatividad } \\
\hline & & $\bar{x}$ & $S$ & $\bar{x}$ & $S$ & $\bar{x}$ & $S$ \\
\hline \multirow{3}{*}{$\begin{array}{c}\text { GR. } \\
\text { CTROL }\end{array}$} & Pretest & 58,31 & 21,806 & 52,02 & 25,390 & 64,97 & 27,799 \\
\hline & Postest & 72,13 & 19,292 & 67,52 & 23,817 & 75,24 & 22,640 \\
\hline & $t$ & \multicolumn{2}{|c|}{$-10,700^{* * *}$} & \multicolumn{2}{|c|}{$-9,307 * * *$} & \multicolumn{2}{|c|}{$-3,802 * * *$} \\
\hline \multirow{3}{*}{$\begin{array}{c}\text { GR. } \\
\text { EXPER. }\end{array}$} & Pretest & 59,64 & 21,793 & 52,32 & 26,468 & 57,82 & 24,016 \\
\hline & Postest & 81,87 & 16,384 & 78,03 & 20,617 & 86,66 & 15,426 \\
\hline & $t$ & \multicolumn{2}{|c|}{$-16,601 * * *$} & \multicolumn{2}{|c|}{$-13,336^{* * *}$} & \multicolumn{2}{|c|}{$-7,095 * * *$} \\
\hline
\end{tabular}

***p $\leq 0,001 \quad * * p \leq 0,01 \quad{ }^{*} p \leq 0,05$ 
Como se puede observar, la significatividad de la $t$ de student en cada una de las dimensiones, tanto para el grupo control como para el experimental, indica que dentro de ambos los niños evolucionan significativamente en todas las áreas de desarrollo. Dicha evolución parece más acusada en los niños del grupo experimental, destacando las diferencias de promedios entre el pretest y el postest correspondiente al desarrollo somático, la conceptualización, la normatividad, la reacción motriz y la coordinación senso-motriz $(28.46,25.71$, $28.84,24.01$ y 24.44 , respectivamente). También evolucionan los niños del grupo control - aunque en menor medida-, particularmente en su desarrollo somático, coordinación senso-motriz y reacción motriz (diferencias de medias entre el pretest y el postest de 18.7, 16.96 y 16.58 respectivamente).

Si consideramos además de su edad, la evolución que manifiestan ambos grupos en cada una de las áreas de desarrollo tenemos que:

- Área socio-afectiva:

- Se aprecia evolución en todos los niños a los que se aplica el método (grupo experimental), muy significativa en niños de 1 y 4 años, cuyo promedio evoluciona en 28.35 y 20.31 puntos respectivamente.

- En cambio, dentro del grupo control sólo manifiestan una evolución significativa los niños de 7 a 12 meses, pero inferior a la del grupo experimental.

- Área Perceptivo-cognitiva (desarrollo somático y senso-perceptivo)

- Los niños del grupo experimental muestran mayores niveles de desarrollo somático y senso-perceptivo - cualquiera que sea su edad - destacando las diferencias de promedios de $39.99,31.17$ y 29.26 en el desarrollo somático correspondiente a los niños con 1, 3 y 2 años respectivamente.

- Dentro del grupo control, salvo en los niños de 1 año, en el desarrollo somático y de 4 años en el desarrollo senso-perceptivo — diferencias de promedios de 28.76 y 20.68, respectivamente-, no se aprecian cambios significativos.

- Área Motriz

- Todos los niños del grupo experimental manifiestan una evolución positiva significativa en la coordinación senso-motriz y la reacción motriz — salvo los niños de 4 años en reacción motriz, cuya evolución no es significativa en nuestra muestraEn particular, destaca la enorme evolución tanto en la reacción como en la coordinación motriz, experimentada por niños de 1 a 3 años, con diferencias de promedios de $35.83,29.77$ y 31.33, 33.08, respectivamente. Así mismo, se constata la evolución en reacción motriz de los niños de 5 años — dif. De promedios $=31.18$ -

- Dentro del grupo control, la evolución más significativa se aprecia también en niños de 1 a 3 años (en torno al 20\%). Los niños de 6 años no manifiestan cambios significativos en ninguna de las dimensiones de este área. De 0 a 6 meses, manifiestan cambios significativos en coordinación, pero no en reacción motriz al contrario de lo que sucede con los niños de 7 a 12 meses.

- Área de Pensamiento y lenguaje (contacto/comunicación; conceptualización/pensamiento; normatividad)

- Una vez más, dentro del grupo experimental se aprecian cambios significativos en todas las dimensiones de este área, en todos los grupos de edad considerados. Par- 
ticularmente, evolucionan en el contacto y la comunicación los niños de 1 año, de 0 a 6 meses y de 3 años (diferencias de promedios de 38.17, 25.3 y 23.9, respectivamente) mientras que en pensamiento destaca la evolución de los niños de 2 y 3 años, (diferencias de promedios respectivas de 32.97 y 25.62) y en la normatividad los cambios significativos de los niños de 6 años, (diferencias de promedios de 35 puntos).

- Los niños del grupo control de 0 a 6 meses y 7 a 12 meses, en la dimensión de contacto y comunicación y 6 años en las dimensiones de pensamiento y normatividad, no manifiestan cambios significativos. La principal evolución en el contacto y comunicación, se aprecia en niños de 1 y 3 años, (diferencias de promedios iguales a 17.93 y 17.63, respectivamente), así como de 4 años en la dimensión de pensamiento y de 5 años en la dimensión de normatividad, (diferencias de promedios iguales a 22.08 y 11.62 , respectivamente).

Una vez descrita la evolución de cada grupo, analizamos el ritmo de desarrollo individual de cada sujeto.

\section{Efectos individuales. Ritmo individual de desarrollo}

Para conocer el ritmo individual de desarrollo de todos los niños, esto es, cuántos manifestaban un desarrollo evolutivo normalizado, acelerado o cierto estancamiento, convertimos las puntuaciones directas de sus cocientes de desarrollo en cada una de las dimensiones de desarrollo, en percentiles, teniendo en cuenta el grupo al que pertenecían - experimental, control- y su edad. Este tipo de puntuaciones nos informa sobre la "posición" de cada niño respecto al conjunto de niños que constituyen su grupo de referencia, de modo que la obtención de percentiles más elevados en el postest, indicaría una mejora en su "posición", un progreso claro en su ritmo de desarrollo. Al calcular el número de niños en los que las diferencias entre las puntuaciones pretest-postest eran negativas, positivas o nulas - en cada grupo y segmento de edad- se obtuvieron diversos resultados de los cuáles exponemos los más relevantes:

- El ritmo de desarrollo perceptivo-cognitivo era estable en niños de 0 a 6 meses, independientemente de que les fuera aplicado el método o no. Ahora bien, en el resto de las áreas, mientras que en los niños del grupo experimental se detecta cierta aceleración, los del grupo control siguen una evolución normativa.

- En niños de 7 a 12 meses, las mejoras más acusadas en el ritmo de desarrollo se dan en el contacto, la comunicación y el desarrollo motriz, en ambos grupos.

- A partir de 1 año - en ambos grupos- disminuye el ritmo de desarrollo senso-perceptivo, mientras se acelera el progreso del desarrollo del contacto y la comunicación sólo en los niños a los que se aplicó el método.

- Los niños de 2 años del grupo experimental, manifiestan progresos en el ritmo de desarrollo de todas las dimensiones, salvo en la senso-perceptiva, que se estabiliza. Sin embargo, en el grupo control se aprecia cierta estabilidad —incluso retrocesos - en el desarrollo socio-afectivo, senso-perceptivo, de reacción motriz y conceptualización.

- Destaca la evolución positiva, en todas las áreas de desarrollo de los niños de 3 años, pertenecientes al grupo experimental. Paralelamente sucede en el grupo control, si 
bien hay que considerar cierta estabilización e incluso estancamiento en el desarrollo somático y la conceptualización.

- En ambos grupos, encontramos niños de 4 años que progresan en el ritmo de desarrollo socio-afectivo y motriz, disminuyendo este ritmo en el desarrollo somático, el contacto y la comunicación.

- Los niños de 5 años, en ambos grupos, mejoran su ritmo de desarrollo socio-afectivo, senso-perceptivo, motriz y de conceptualización.

- En niños de 6 años, es de destacar el aumento en el ritmo de desarrollo del lenguaje, en los niños del grupo experimental, no apreciable en los del grupo control — se estabilizan-.

\section{Efectos entre los grupos. Eficacia del método para promover el desarrollo}

Para comprobar si la aplicación del Método produce efectos significativos de mejora en las distintas áreas de desarrollo, comparamos las puntuaciones que obtuvieron los niños del grupo experimental con las de los niños del grupo control, en cada una de las dimensiones de desarrollo. Pero previamente, para verificar si ambos grupos eran equivalentes inicialmente o existía la influencia de otros factores - por ejemplo, vinculados a la selección de los sujetos - se compararon sus promedios en el pretest, mediante la $t$ de Student. Como se aprecia en la tabla 3 (pretest), en ninguna dimensión de desarrollo aparecen diferencias significativas - al nivel de significación de 0,05- entre los promedios del grupo experimental y el de control, antes de aplicar el método. Ello permite suponer que ambos grupos eran equivalentes al principio.

Respecto a la hipótesis general de que los niños pertenecientes al grupo experimental obtendrían resultados significativamente superiores a los del grupo control, por haber recibido la estimulación del método, tal como se observa en la tabla 3 (postest), la diferencia de promedios - pretest-postest - resulta ser claramente significativa en todas las dimensiones de desarrollo. Por tanto, los niños del grupo experimental mejoraron significativamente sus niveles de desarrollo en todas las áreas, respecto al grupo control.

En particular, se constata la siguiente evolución positiva global de promedios de desarrollo: en el área socio-afectiva de un cociente de desarrollo del 58,91\% al 75,66\%; en el área perceptivo-cognitiva, del 52,79\% al 81,25\% — desarrollo somático-y del 68,34\% al $87,11 \%$ - desarrollo senso-perceptivo — ; en el área motriz, del 59,47\% al 83,48\% — reacción motriz-y del $61,90 \%$ al $86,34 \%$ - coord. Sensomotriz-; en el área de pensamiento y lenguaje, del $59,64 \%$ al $81,87 \%$ - contacto y comunicación-, del $52,32 \%$ al $79,03 \%$ - conceptualización-y del $57,82 \%$ al $86,66 \%$ - normatividad-. Las medias de cambio - expresadas en porcentaje - en cada una de las dimensiones, tanto para el grupo experimental como para el grupo control, se muestran en el siguiente gráfico (ver figura 5). Así, los mayores cambios se aprecian en las áreas de pensamiento y lenguaje y en el desarrollo somático.

Sin embargo, si calculamos de nuevo la $t$ de Student para ver las diferencias de medias entre ambos grupos, teniendo en cuenta la edad de los niños, observamos los siguientes resultados: en todas las áreas de desarrollo cualquiera que sea su edad, aquellos que pertenecen al grupo experimental obtienen promedios más elevados que los del grupo control, aho- 
TABLA 3: Efectos del Método entre los Grupos ( $t$ de Student).

\begin{tabular}{|c|c|c|c|c|c|c|c|c|c|c|c|}
\hline \multicolumn{12}{|c|}{ Efectos entre-grupos } \\
\hline \multirow{2}{*}{\multicolumn{2}{|c|}{$\begin{array}{l}\text { Cociente de } \\
\text { Desarr. }\end{array}$}} & \multicolumn{2}{|c|}{ Afectividad } & \multicolumn{2}{|c|}{ Somático } & \multicolumn{2}{|c|}{ Senso-perc. } & \multicolumn{2}{|c|}{ R. Motriz } & \multicolumn{2}{|c|}{ C. Senso-Mot } \\
\hline & & $\bar{x}$ & $S$ & $\bar{x}$ & $S$ & $\bar{x}$ & $S$ & $\bar{x}$ & $S$ & $\bar{x}$ & $S$ \\
\hline \multirow{3}{*}{ Pretest } & $\begin{array}{l}\text { Gr. } \\
\text { Ctrol }\end{array}$ & 57,69 & 23,125 & 53,15 & 25,871 & 65,23 & 28,612 & 55,38 & 25,145 & 60,09 & 24,481 \\
\hline & $\begin{array}{l}\text { Gr. } \\
\text { Exp. }\end{array}$ & 58,91 & 22,501 & 52,79 & 25,156 & 68,34 & 25,810 & 59,47 & 54,621 & 61,90 & 23,493 \\
\hline & $t$ & \multicolumn{2}{|c|}{$-0,587$} & \multicolumn{2}{|c|}{0,156} & \multicolumn{2}{|c|}{$-1,249$} & \multicolumn{2}{|c|}{$-1,051$} & \multicolumn{2}{|c|}{$-0,823$} \\
\hline \multirow{3}{*}{ Postest } & $\begin{array}{c}\text { Gr. } \\
\text { Ctrol }\end{array}$ & 68,68 & 20,582 & 71,85 & 22,461 & 78,59 & 23,514 & 71,96 & 22,941 & 76,91 & 19,580 \\
\hline & $\begin{array}{l}\text { Gr. } \\
\text { Exp. }\end{array}$ & 76,66 & 20,529 & 81,25 & 19,072 & 87,11 & 19,743 & 83,48 & 21,224 & 86,34 & 16,742 \\
\hline & $t$ & \multicolumn{2}{|c|}{$-3,712 * * *$} & \multicolumn{2}{|c|}{$-4,929 * * *$} & \multicolumn{2}{|c|}{$-4,289 * * *$} & \multicolumn{2}{|c|}{$-5,698 * * *$} & \multicolumn{2}{|c|}{$-5,659 * * *$} \\
\hline
\end{tabular}

\begin{tabular}{|c|c|c|c|c|c|c|c|}
\hline \multirow{2}{*}{\multicolumn{2}{|c|}{ Cociente de Desarr. }} & \multicolumn{2}{|c|}{ Contacto-Comunica. } & \multicolumn{2}{|c|}{ Conceptualización } & \multicolumn{2}{|c|}{ Normatividad } \\
\hline & & $\bar{x}$ & $S$ & $\bar{x}$ & $S$ & $\overline{\boldsymbol{x}}$ & $S$ \\
\hline \multirow{3}{*}{ Pretest } & Gr. Ctrol & 58,31 & 21,806 & 52,02 & 25,390 & 64,97 & 27,799 \\
\hline & Gr. Exp. & 59,64 & 21,793 & 52,32 & 26,468 & 57,82 & 24,016 \\
\hline & $t$ & \multicolumn{2}{|c|}{$-0,665$} & \multicolumn{2}{|c|}{$-0,099$} & \multicolumn{2}{|c|}{1,201} \\
\hline \multirow{3}{*}{ Postest } & Gr. Ctrol & 72,13 & 19,292 & 67,52 & 23,817 & 75,24 & 22,640 \\
\hline & Gr. Exp. & 81,87 & 16,384 & 78,03 & 20,617 & 86,66 & 15,426 \\
\hline & $t$ & \multicolumn{2}{|c|}{$-5,949 * * *$} & \multicolumn{2}{|c|}{$-4,120 * * *$} & \multicolumn{2}{|c|}{$-2,570^{*}$} \\
\hline
\end{tabular}

*** $p \leq 0,001 \quad * * p \leq 0,01 \quad * p \leq 0,05$

ra bien, estas diferencias en algunos casos no son tan amplias como para resultar significativas al nivel de significación 0,05 , de ahí que destaquemos los siguientes resultados:

- Dentro del área socio-afectiva, resulta muy significativa la evolución en los niños de 0 a 6 meses — su cociente de desarrollo pasa del 73,95\% al 91\%—, de 2 años — del $60,92 \%$ al $71,05 \%$ - y de 3 años — del 67,29\% al 76,94\%-.

- Dentro del área perceptivo-cognitiva, se encuentran cambios significativos en el desarrollo somático de los niños de 0 a 6 meses — del 58,5\% al 89,15\% - de 7 a 12 meses — del 58,95\% al 79,25\%—, 3 y 4 años — del 73,71\% al 80,92\% y del 77,93\% al $85,57 \%$, respectivamente- y en el desarrollo senso-perceptivo de niños de 2 y 4 años — de 67,24\% al 79,84\% y del 85,84\% al 95,21\%, respectivamente-

- Dentro del área motriz, resultan muy significativos los cambios en reacción motriz experimentados por niños de 0 a 6 meses — del 63,1\% al 86,68\%- 1 año - del 


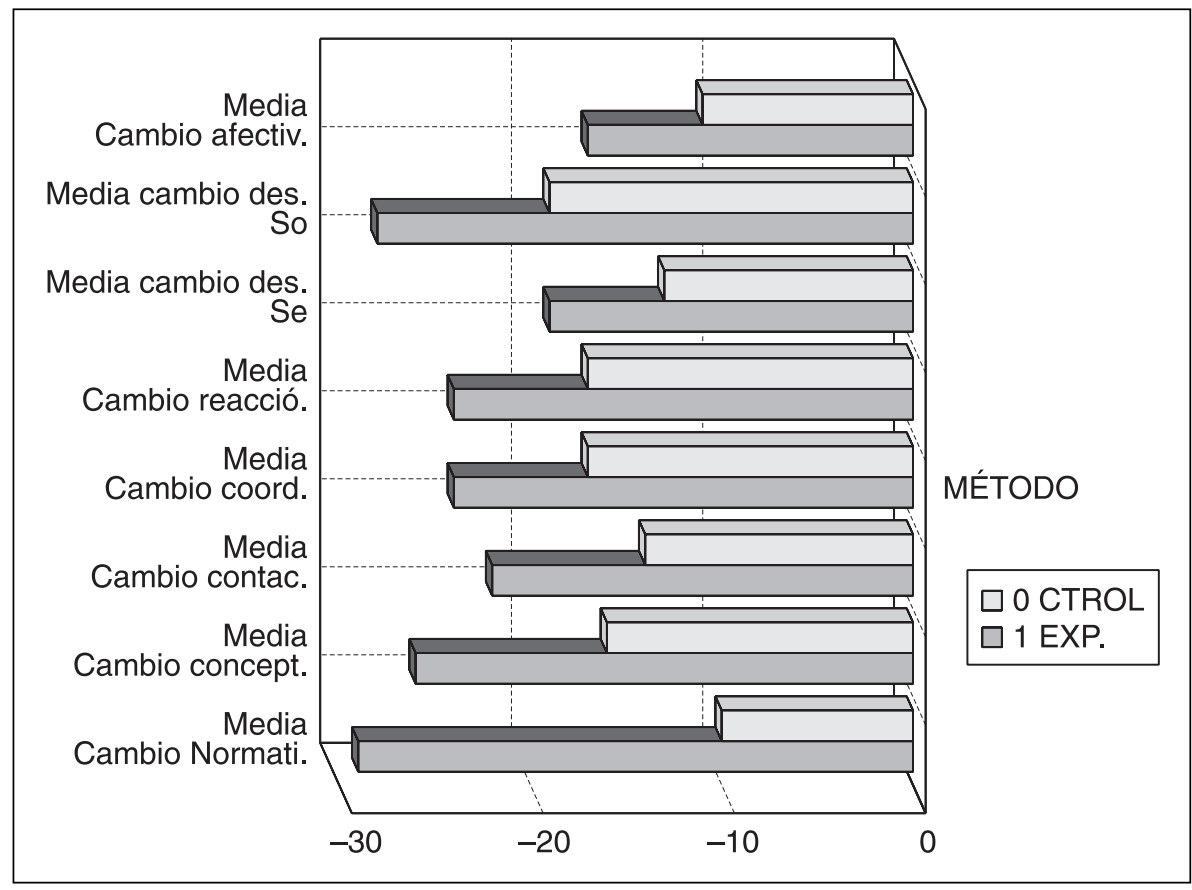

FIGURA 5.

Cambios en las dimensiones de desarrollo, debidos al método.

$75,36 \%$ al 91,58\% - y 3 años — del 68,83\% al 82,1\% - y en coordinación senso-motriz, por niños de 0 a 6 meses — del 70,02\% al 85\%-, 2, 3 y 4 años — del 76,13\% al $87,55 \%$, del 70,58\% al 84,27\% y del $85,08 \%$ al $92,2 \%$, respectivamente-.

- Dentro del área de pensamiento y lenguaje, destaca la evolución muy significativa del contacto y la comunicación en niños de 0 a 6 meses, 2, 3, 4, 5 y 6 años - con incrementos de promedios del $15,9 \%, 10,12 \%, 10,94 \%, 7,67 \%, 6,97 \%$ y $13,09 \%$ respectivamente- y de la conceptualización en niños de 3 y 4 años — con incrementos de promedios del $12,33 \%$ y $9,34 \%$ -

Resumiendo, en los niños de 0 a 6 años, los cambios de desarrollo más significativos se manifiestan en las áreas socio-afectiva, motriz y desarrollo somático, contacto y comunicación. Los niños de 7 a 12 meses, evolucionan significativamente en su desarrollo somático, así como los de 1 año en sus reacciones motrices. Los niños de 2 años manifiestan cambios significativos en su desarrollo socio-afectivo, senso-perceptivo, senso-motriz, contacto y comunicación. Los niños de 3 años, evolucionan significativamente en las áreas socio-afectiva, motriz, desarrollo somático, contacto, comunicación y conceptualización, mientras que los niños de 4 años mejoran significativamente en el área perceptivo-cognitiva, motriz y de pensamiento y lenguaje. Finalmente, los niños de 5 y 6 años evolucionan significativamente en el contacto y la comunicación. 
Por otra parte, aunque los resultados anteriores den respuesta a la mayor parte de las cuestiones planteadas en nuestro estudio, llevamos a cabo otro tipo de análisis complementarios con el propósito de recoger más evidencias sobre el efecto del Método de estimulación, en los niños del grupo experimental. Así, seguimos las recomendaciones de algunos autores, (Ato, 1995), quiénes reconocen que no existe acuerdo en la forma de practicar un análisis óptimo con este tipo de datos — pretest-postest - pero, en cualquier caso, se puede estimar el efecto del método ajustando las diferencias iniciales —en el pretest - entre ambos grupos, así como otras amenazas contra la validez interna derivadas de las comparaciones intrasujeto, a través de un análisis de covarianza que considerase el Pretest como covariante y analizando la existencia de efectos de interacción entre el Pretest y el método.

Nuevamente, con ayuda del programa SPSS, tras realizar diversos análisis que permitiesen elegir el modelo más adecuado de ANCOVA a partir de los supuestos estadísticos que cumplían nuestros datos, llegamos a diversas soluciones. Para no extendernos excesivamente sólo se presentan los datos estadísticos correspondientes al efecto principal del método (ver Tabla 4) aunque, a continuación, se comentan también los efectos debidos al nivel inicial de desarrollo — pretest - y la influencia de la edad.

TABLA 4: Principales resultados del Análisis de Covarianza correspondientes al efecto principal del Método.

\begin{tabular}{|l|r|r|c|}
\hline \multicolumn{1}{|c|}{ Dimensión } & F método & Sig. & $\begin{array}{c}\text { Efecto: Eta al } \\
\text { cuadrado parcial }\end{array}$ \\
\hline Afectividad (Af) & 226,154 & 0,000 & 0,448 \\
\hline Desarrollo Somático (som) & 33,420 & 0,000 & 0,068 \\
\hline Desarrollo Senso-perceptivo (sens) & 19,365 & 0,000 & 0,04 \\
\hline Reacción Motriz (mot) & 39,043 & 0,000 & 0,078 \\
\hline Coordinación Senso-Motriz (sm) & 475,279 & 0,000 & 0,668 \\
\hline Contacto y Comunicación (com) & 39,163 & 0,000 & 0,078 \\
\hline Conceptualización (cpto) & 232,097 & 0,000 & 0,598 \\
\hline Normatividad (nor) & 26,364 & 0,000 & 0,271 \\
\hline
\end{tabular}

Así, respecto al área socio-afectiva, el modelo de ANCOVA explica el 94,1\% de la variabilidad de este área en el postest, de modo que las diferencias de afectividad que, al cabo de cuatro meses de aplicación del método, manifiestan los niños del grupo experimental, sí resultan significativas, teniendo en cuenta su nivel inicial en este área. Dicho nivel inicial - el pretest - influye significativamente en la evolución que manifiestan, independientemente del efecto de edad — maduración-, pues ésta última, por sí sola no es capaz de explicar significativamente los progresos de desarrollo detectados. En cambio, no se encuentran diferencias significativas en afectividad entre el grupo experimental y el de control en los sub-grupos establecidos según su edad y nivel afectivo inicial o el efecto de interacción entre la edad y el método. 
Respecto al tamaño del efecto tratamiento, "Eta al cuadrado parcial" indica la proporción de varianza de la afectividad en el postest - Variable dependiente - que es capaz de explicar el método por sí solo, en nuestro caso, un 48,8\% de variabilidad. En consecuencia, la aplicación del método explica prácticamente la mitad de los cambios en desarrollo socio-afectivo, que experimentan los niños del grupo experimental.

En el área perceptivo-cognitiva, la aplicación del método produce diferencias significativas - a favor del grupo experimental - tanto en el desarrollo somático como en el senso-perceptivo, cuyos modelos de ANCOVA explican, respectivamente, el 94,8\% y el $96,3 \%$ de su variabilidad. Además, se han encontrado diferencias significativas tanto en el desarrollo somático como en el senso-perceptivo, por efecto - independiente y conjunto- de la edad y del nivel inicial de los niños en estas dimensiones de desarrollo - pretest-.

Por otra parte, el tamaño del efecto del método en el desarrollo somático es del $6,8 \%$, mientras que en el desarrollo senso-perceptivo es del $4 \%$.

En cuanto al área motriz, nuevamente el método produce diferencias significativas a favor del grupo experimental, tanto en la reacción motriz - donde el modelo explica el 94,6\% de varianza- como en la coordinación senso-motriz - dónde se explica el 96,3\% de varianza-. Además, se aprecian diferencias significativas entre los grupos establecidos según la edad, el nivel previo de reacción motriz y la interacción entre ambos factores. Paralelamente, el nivel previo de coordinación senso-motriz también influye significativamente en los resultados obtenidos tras la aplicación del método, llegando a interactuar significativamente con este. Finalmente, en cuanto al tamaño de los efectos del método, éste contribuye a explicar el 7,8\% de la variabilidad de la reacción motriz y más de la mitad de la variabilidad de la coordinación senso-motriz, el 66,8\%.

En el área de pensamiento y lenguaje, también se observan diferencias significativas - tras la aplicación del método- en los niveles de desarrollo de todas sus dimensiones, superiores en los niños del grupo experimental. Específicamente, los modelos de ANCOVA considerados explican el $96,4 \%, 94,5 \%$ y el $97,2 \%$ de la varianza de las dimensiones de contacto y comunicación, conceptualización y normatividad, respectivamente. Además de las diferencias significativas que el método produce en el contacto y comunicación, se aprecian diferencias debidas al nivel inicial en esta dimensión, la edad y la interacción entre ambos factores. Paralelamente, el método resulta especialmente efectivo en la explicación del nivel de conceptualización alcanzado en el postest por los niños del grupo experimental — superior al de control-, moderado por la influencia de su nivel inicial de desarrollo, circunstancia que también sucede en el desarrollo de la normatividad. El tamaño del efecto del método es del 7,8\% en el desarrollo del contacto y la comunicación, del 59,8\% en el de la conceptualización y del $27,1 \%$ en la normatividad.

Finalmente, para contrastar las hipótesis de relación entre las edades de padres y el nivel de desarrollo alcanzado en las distintas áreas, calculamos diversos coeficientes de correlación - Pearson, Spearman - entre estas variables. A un nivel de 0,05 de significación, globalmente, no parecen existir relaciones significativas entre las edades de los padres y cada una de las dimensiones del desarrollo. Tampoco se han encontrado - en esta muestra - correlaciones significativas $-\alpha=0,05$ - entre el número de hermanos/as y los niveles de desarrollo alcanzados en las distintas áreas. 


\section{Discusión de resultados y conclusiones}

La eficacia del Método Estitsológico Multisensorial ha quedado demostrada en la muestra considerada (así como también lo fue en estudios sobre madres y niños en la cárcel) ya que su aplicación ha producido diferencias significativas en el desarrollo general de los niños del grupo experimental, en comparación con el alcanzado por el grupo control.

Al estudiar la evolución interna de estos grupos - efectos dentro de los grupos - se ha podido constatar un importante efecto de maduración, por el cual ambos evolucionan en todas las dimensiones de desarrollo, (especialmente en el desarrollo somático), si bien, es mayor el ritmo de desarrollo que experimentan los niños del grupo experimental, particularmente en la conceptualización y en la normatividad.

Al tener en cuenta la edad, se constatan los efectos significativos del método en todos los niños del grupo experimental que produce mejoras en todas las dimensiones de desarrollo (menos acusadas en el desarrollo afectivo de los niños de 7-12 meses cuya afectividad inicial era particularmente elevada). En algunos casos esta evolución interna se aprecia paralelamente en ambos grupos como el aumento del desarrollo socio-afectivo en niños de 1 a 4 años, del desarrollo somático en niños de 1 año, senso-perceptivo en niños de 4 años, el área motriz en niños de 3 años y el contacto y la comunicación en niños de 3 años. Sin embargo, en otras ocasiones, los niños del grupo control no manifiestan cambios significativos que sí se aprecian en el grupo experimental, como en el área perceptivo-cognitiva, motriz, contacto y comunicación, en niños con edades inferiores al año. En consecuencia, con el transcurso del tiempo, en ambos grupos se constatan progresos evolutivos más generalizados en los niños del grupo experimental.

Respecto al ritmo de desarrollo individual de los niños del grupo experimental, en general, en niños de todas las edades tiende a acelerarse en la mayor parte de las áreas de desarro1lo, en algunos casos estas mejoras se acusan sólo en los niños a los que se les aplicó el método mientras que en los niños del grupo control se estabilizaba el ritmo (como en todas las dimensiones en niños de 3 años. En otros casos se aprecian progresos en el ritmo de desarrollo de ambos grupos (en el contacto, comunicación y desarrollo motriz en niños de 7 a 12 meses; el desarrollo socio-afectivo y motriz en niños de 4 y 5 años etc.). Por tanto, en términos generales es mayor la mejora del ritmo individual del desarrollo en los niños del grupo experimental, si bien dentro del grupo control también se detectan progresos evolutivos más susceptibles a las etapas de estancamiento e incluso retroceso aparente inherentes al concepto de Desarrollo que, como proceso, no alcanza su estadío final de una vez, sino progresivamente.

Comprobada la equivalencia inicial entre los grupos — además del efecto madurativola $t$ de Student muestra la existencia de efectos significativos causados por el método, en el grupo experimental, en todas las dimensiones de desarrollo y, especialmente, en el somático, el pensamiento y el lenguaje. Incluso al tener en cuenta la edad cronológica, los promedios del grupo experimental superan en todas las áreas a los del grupo control, aunque no siempre resulten significativas estas diferencias. Así, en algunos intervalos de edad, la eficacia del método se extiende a más dimensiones de desarrollo, como en el caso de niños de 0 a 6 meses, 2 y 3 años — socio-afectivo, percepto-cognitivo, motriz, pensamiento y lenguaje - mientras que en otras edades, dicha efectividad parece más focalizada — el desarrollo perceptivo-cognitivo en niños de 7 a 12 meses y 4 años, motriz en niños de 1 año y de pensamiento-lenguaje a los 5 y 6 años-. 
Por otra parte, los análisis de ANCOVA realizados confirman nuevamente su eficacia, especialmente en el desarrollo socio-afectivo, la coordinación senso-motriz y el pensamiento, con porcentajes de influencia significativa muy elevados — aproximadamente del $50 \%$ al 70\%-, también relevantes en el resto de las áreas — del 4\% al 8\% - que justifican su aplicación como estrategia eficaz de estimulación temprana.

$\mathrm{Al}$ analizar las influencias de la edad, los niveles de desarrollo previos y el método, tomados como variables aisladas y en interacción, se aprecia que el método es el principal factor explicativo de las mejoras sobre todo en los niveles de desarrollo socio-afectivo, la Coordinación Senso-Motriz y conceptualización, el contacto y la comunicación -aunque en esta última dimensión también fuera relevante el nivel previo- - La edad de los niños y los niveles de desarrollo inicial resultan fundamentales para explicar la variabilidad, especialmente en el caso del Desarrollo Somático, Senso-perceptivo, la Reacción Motriz y la Normatividad. Por ejemplo apreciamos más diferencias en el Desarrollo Somático, entre el grupo experimental y el grupo control, en el $1^{\text {er }}$ año de vida - de 0 a 6 meses y de 7 a 12 meses - que en otros intervalos de edad.

Así mismo, el método interactúa con los niveles previos de desarrollo en algunas dimensiones, fundamentalmente en la coordinación senso-motriz en niños de 1 y 6 años, en el pensamiento en niños de 6 años y la normatividad en niños de 5 y 6 años. En estos casos, además de mejoras, parece tener un efecto compensador de los déficits que inicialmente pudieran presentar los niños en estas dimensiones, aspecto a explorar en futuras investigaciones.

Finalmente, las hipótesis que vinculaban los niveles de desarrollo en las diversas áreas con la edad de los padres y el número de hermanos, han debido rechazarse, ya que no parecen existir en general - al menos en esta muestra-, relaciones significativas entre ambos. Tal vez el hecho de que los padres de mayor edad tengan niños cuyo desarrollo socioafectivo sea menor, pueda explicarse por una menor tendencia a compartir o permitir juegos que conlleven algún riesgo, prevenir accidentes - por ejemplo coartando el uso de toboganes u oros aparatos en el parque... - pero es un aspecto que convendría contrastar también en futuras investigaciones.

En conclusión, se ha mostrado la eficacia del Método Estitsológico Multisensorial al aplicarlo, durante cuatro meses, a niños de 0 meses a 6 años, sin alteraciones o discapacidades manifiestas, procedentes de contextos normalizados. Teniendo en cuenta la brevedad del período de tratamiento y las dificultades metodológicas que se derivan de la propia heterogeneidad - característica de los niños de estas edades - así como la elevada variabilidad inherente a los procesos de desarrollo, puede afirmarse que los resultados son satisfactorios y justifican su aplicación. No obstante, son recomendables futuras investigaciones sobre la eficacia del Método Estitsológico Multisensorial para mejorar el desarrollo de los niños, en distintos contextos y también con alteraciones específicas detectadas tanto en niños como en contextos.

\section{Referencias bibliográficas}

Alfonso, J. M. e Ibáñez, P. (1987). Las minusvalías: diagnóstico, tratamiento e integración. Madrid: UNED.

Anguera, Ma . T. y otros (1995). Métodos de investigación en Psicología. Madrid: Síntesis. Anguera. 
Ato, M. (1995). “Análisis estadístico I: Diseños con variable de asignación no conocida”. En Anguera, Ma. T. y otros, Métodos de investigación en Psicología (pp. 271-304). Madrid: Síntesis.

Bowlby, J. (1976). El vínculo afectivo. Buenos Aires: Paidós.

Brazelton, T. B. (1974). "The origins of reciprocity: the early mother infant interaction". In Lewis, M., The effects of th infant on its caregiver. New York: Silley.

Brofenbrenner, U. (1975). “Is Early intervention effective?”. En M. Gttentag \& E. Streuning (comps.), Handbook of evaluation research, vol 2. London: Sage Publications.

Dueñas, M. L. (2002). "Importancia de la inteligencia emocional: un nuevo reto para la orientación educativa". Educación $X_{1}$ : Revista de la Facultad de Educación, 5, 77-96.

Heward, W. (2000). Niños excepcionales ( $5^{\mathrm{a}}$ Ed.). Madrid: Prentice Hall.

Ibáñez, P. (2003). Potencie la inteligencia de su hijo. Madrid: Dykinson.

Ibáñez, P. (2002). Las discapacidades: Orientación e Intervención Educativa. Madrid: Dykinson.

Ibáñez, P. y Alfonso, C. (1999). "Incidence of Affectivity in the Development of Babies". Singapur: World Organization for Early Childhood Education.

Ibáñez, P. y Senra, M. (1999 a). "Análisis de una experiencia de estimulación multisensorial con el Método Estitsológico". Educación XXI, 2 (2), 265-276. Madrid: UNED, Facultad de Educación.

Ibáñez, P. y Senra, M. (1999 b). "La afectividad en las madres reclusas". Revista Española de Orientación y Psicopedagogía, (AEOP), 10 (17), 227-236.

Ibáñez, P. (1997). Aumente la inteligencia de su hijo. Madrid: Dykinson.

Ibáñez, P. (1987). Programación y evaluación para educación especial. Madrid: UNED.

Naciones Unidas (2002). Un mundo apropiado para los niños y las niñas. UNICEF: New York.

Real Patronato sobre Discapacidad. (2003, $5^{\mathrm{a}}$ reimp.). Libro Blanco de la Atención Temprana. Documentos 55/2000.Madrid: Real Patronato sobre Discapacidad.

Reichardt, C. A. (1979). "The statistical analysis from non-equivalent group designs". En T. D. Cook y D. T. Campbell, Quasi-experimentation: Design and Analysis issues for field settings (pp. 147205). San Francisco, CA: Jossey-Bass.

Salmeron, P. (2002). "Evolución de los conceptos sobre inteligencia. Planteamientos actuales de la inteligencia emocional para la orientación educativa". Educación $X X_{1}$ : Revista de la Facultad de Educación, 5, 97-122.

Secadas, F. (1988). Escala Observacional del Desarrollo. Madrid: Tea.

Spizt, H. H. (1979). El primer año de la vida del niño. Madrid: Aguilar.

Fecha de recepción: 24-01-04

Fecha de revisión: 15-07-04

Fecha de aceptación: 20-04-05 